\title{
Sensitivity Analysis of a Simplified Fire Dynamic Model
}

\author{
Lars Schiott Sorensen and Anker Nielsen \\ Danish Building Research Institute, Aalborg University, Copenhagen DK-2450, Denmark
}

\begin{abstract}
This paper discusses a method for performing a sensitivity analysis of parameters used in a simplified fire model for temperature estimates in the upper smoke layer during a fire. The results from the sensitivity analysis can be used when individual parameters affecting fire safety are assessed. If the variation of a single parameter is found to have a major impact on fire safety, it may be necessary to conservatively select this parameter in order to incorporate additional safety. We compare fire scenarios in rooms surrounded by lightweight as well as heavy walls in order to investigate which parameters are the most significant in each case. We apply the Sobol method, which is a quantitative method that gives the percentage of the total output variance that each parameter accounts for. The most important parameter is found to be the energy release rate that explains $92 \%$ of the uncertainty in the calculated results for the period before thermal penetration $\left(t_{P}\right)$ has occurred. The analysis is also done for all combinations of two parameters in order to find the combination with the largest effect. The Sobol total for pairs had the highest value for the combination of energy release rate and area of opening, which explains $96 \%$ of the uncertainty. After thermal penetration, the energy release rate is still the most important parameter, but now only explains $49 \%$ of the variation. The second parameter is the thickness of the surface material, which explains $43 \%$.
\end{abstract}

Key words: Sensitivity analysis, fire model, heavy walls, lightweight walls, fire-room geometry.

\section{Introduction}

Fire modeling is often done without a proper following sensitivity analysis. A lack of a sensitivity analysis, or a bad choice of input data, is often seen when fire consultants are modeling fires. It seems that the problem is partly due to a lack of knowledge on the field and an absence of supporting research about the most important parameters. This study aims to establish a better understanding of the most significant input parameters to be analyzed during a sensitivity study. We performed the analysis based on a simplified energy balance valid for pre-flashover situations.

Input data in fire-safety calculations are subject to greater or lesser uncertainties. At the same time, uncertainties appear in the applied calculation models, among other things, due to a larger or smaller number of assumptions and simplifications of reality.

The purpose of a sensitivity analysis is to identify

Corresponding author: Lars Schiott Sorensen, Ph.D., senior researcher, research field: fire-safety engineering. E-mail: LSS@sbi.aau.dk. which parameters have the most influence on the calculation results and then assess whether the values of these parameters are chosen with sufficient certainty.

The purpose is to find out how much it means for the results if you choose, for instance, an energy release rate that is twice as big as first assumed, i.e.:

$$
\frac{\Delta T}{\Delta \dot{Q}}, \frac{\Delta z}{\Delta \dot{Q}}, \frac{\Delta Y}{\Delta \dot{Q}}
$$

where, $\Delta T$ is variation of temperature, $\Delta z$ is variation of smoke layer, $\Delta Y$ is variation of gas concentration and $\Delta \dot{Q}$ is variation of energy release rate.

Therefore, variation or sensitivity is found respectively for temperature, smoke layer thickness and the concentration of gases caused by a variation in the maximum energy release rate [1].

Parameters are determined in the fire-strategy report, which include fire location, growth factor, maximum energy release rate, the number of usable exits, etc., and which should be subjected to a sensitivity analysis.

The deterministic analysis provides useful 
information about the fire-safety impact, but because of the uncertainty of the calculation models and input data, a sensitivity analysis should always be carried out.

The uncertainties that exist in connection with a deterministic analysis and make it important to conduct a sensitivity analysis, are, for instance, the following [1]:

- Uncertainties arising from the qualitative construction review presented in the fire-strategy report. It can, for example, be the number of people in the building and where they are located, etc.;

- Selected simplifications made for the sake of the calculation method used. It can, for instance, be the use of a two-zone model that is a simplification of a 3D situation, or a dome that has been reduced to a cube in order to fit into the model in the computer simulation;

- Selected fire scenarios, including fire growth rate and maximum energy release rate. An analysis should always be performed on the effect of an altered growth rate and a modified maximum energy release rate, as well as another location. For example, it also has a major influence on the results whether a door to a stairwell is closed or open in the designed scenario, because an open door can have a great effect. In such cases, the risk of the door being open should be closely examined. In most cases, a series of simulations should be conducted on a number of fire scenarios with doors open/closed, fast and slow fire growth, greater and lesser energy release rates;

- Assumptions about the reliability of the active and passive systems. A sensitivity analysis should be carried out to assess what should be done if, for example, a sprinkler system is not working as intended. This will, for instance, determine that the system is not operational or only partially controls the fire. If separating building elements, doors and penetrations, etc., fail, the impact should also be examined;

- Evacuation conditions. Similarly, a sensitivity analysis should be performed for evacuation, where, for example, a door is blocked, or where the number of people has increased relative to what was assumed.

A measure of the sensitivity of the selected design is the difference between the results obtained by changing individual parameters.

A sensitivity analysis is usually limited to the most critical situations.

The results from a sensitivity analysis can be used when individual parameters affecting fire safety are assessed. If the variation of a single parameter is found to have a major impact on fire safety, it may be necessary to conservatively select this parameter in order to incorporate additional safety. Similarly, it may turn out that extra safety is needed if a fire safety system, e.g., a sprinkler system, turns out to be crucial for fire safety. A detailed assessment of this may take place after a risk analysis.

The result of a sensitivity analysis can tell us whether the inequality:

$$
t_{\text {crit }}>t_{\text {evac }}
$$

is still satisfied after a change in an input data parameter has taken place. $t_{c r i t}$ is the time until a critical condition arises (could be the temperature) and $t_{\text {evac }}$ is the total evacuation time for all people in the building.

Then how is $t_{c r i t}$ assessed against the evacuation time $t_{\text {evac }}$ ? It is a difficult issue as there is probably no conclusive answer. However, the level of personal safety is determined by the following inequality:

$$
t_{c r i t}>t_{w}+t_{r d}+t_{g}=t_{\text {evac }}
$$

where, $t_{w}$ is warning time, $t_{r d}$ is reaction and decision time, $t_{g}$ is flow and walking time.

But how much longer than $t_{\text {evac }}$ should $t_{c r i t}$ be? A safety factor $(S F)$ can be incorporated as:

$$
t_{\text {crit }}>S F \cdot t_{\text {evac }}
$$

This principle is analogue to the partial coefficient method used in statics. The safety factor is used by different countries with performance-based codes among others, such as New Zealand and Sweden. In New Zealand, $S F$ is selected with the value of 2 for ordinary people who are able to reach safety by themselves. A greater value is recommended for the 
disabled and the elderly. The safety factor is used to account for the uncertainties in the calculation of both $t_{c r i t}$ and $t_{\text {evac }}$. Problems with finding the way and other unforeseen events may occur that affect the evacuation time. Similarly, the calculation of the time until critical conditions can be subject to large errors, for example, caused by an inappropriate choice of models, incorrect calculation assumptions or uncertain defined values of the input data.

Another approach to the problem could be simply to look at the numerical difference between $t_{c r i t}$ and $t_{\text {evac. }}$.

That is to say, first and foremost, an assessment of $\Delta t$ defined as:

$$
\Delta t=t_{\text {evac }}-t_{\text {crit }}
$$

This value must be less than 0 in order to proceed with the calculation procedure. In other words:

$$
\Delta t<0
$$

If $|\Delta t|$ is small, a risk analysis should be performed, since a small numerical value indicates that even the slightest sensitivity to an incorrectly chosen parameter may be decisive for the outcome. Therefore, a risk analysis is suitable for obtaining a clearer picture of the events that can occur during a fire scenario with a certain probability and associated impacts.

A schematic sequence for the calculation procedure of the last-described procedure is shown in Fig. 1 [1].

\section{Model Theory}

For our sensitivity analysis, we consider a fire model for estimating the temperature of smoke gas prior to flashover. The fire has typically divided the room volume in a cold lower zone and a hot upper one. Therefore, we often use a two-zone model to describe the situation.

Energy and mass balances must be met in order to obtain correct results.

As the background for sensitivity analysis, we use a simplified energy balance, established by McCaffrey et al. [2]:

$$
\left[\begin{array}{l}
\text { Energy } \\
\text { release } \\
\text { rate }
\end{array}\right]=\left[\begin{array}{l}
\text { Loss } \\
\text { through } \\
\text { openings }
\end{array}\right]+\left[\begin{array}{l}
\text { Loss } \\
\text { through } \\
\text { surroundings }
\end{array}\right]
$$

which can be written as:

$$
\dot{Q}=\dot{m}_{g} \cdot c_{p} \cdot\left(T_{g}-T_{a}\right)+\dot{q}_{\text {loss }}
$$

where, $\dot{Q}$ is energy release rate $(\mathrm{kW}), \dot{m}_{g}$ is rate of gasification $(\mathrm{kg} / \mathrm{s}), c_{p}$ is the specific heat capacity $(\mathrm{kJ} / \mathrm{kg}), T_{g}$ is the gas temperature $(\mathrm{K}), T_{a}$ is the ambient temperature $(\mathrm{K})$ and $\dot{q}_{\text {loss }}$ is heat loss to surroundings $(\mathrm{kW})$.

Utilizing a range of fire dynamic expressions as well as experiments, and statistical correlation, McCaffrey stated the following expression for the temperature rise $\Delta T$ which is equal to $\left(T_{g}-T_{a}\right)$ in Eq. (7):

$$
\Delta T=6.85\left(\frac{\dot{Q}^{2}}{A_{o} \sqrt{H_{o}} \cdot h_{k} \cdot A_{T}}\right)^{1 / 3}
$$

where,

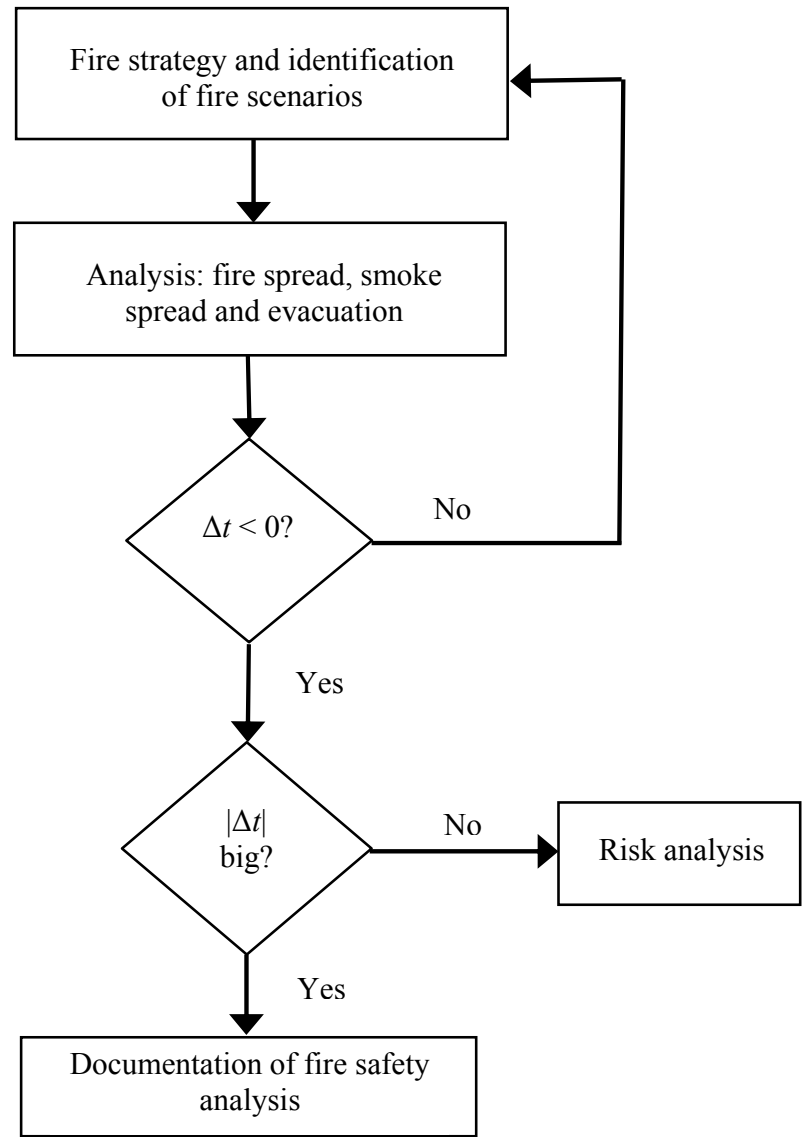

Fig. 1 Schematic representation of the calculation procedure, including a sensitivity analysis. 
$h_{k}$ : effective heat exchange number $\left(\mathrm{kW} / \mathrm{m}^{2} \mathrm{~K}\right)$;

$A_{o}$ : opening area $\left(\mathrm{m}^{2}\right)$;

$A_{T}$ : area of floors, walls and ceilings (excluding opening area) $\left(\mathrm{m}^{2}\right)$;

$H_{o}$ : opening height $(\mathrm{m})$.

If we ignore the cooling effect of the surfaces, equalize surface temperature $T_{s}$ and $T_{g}$, and assume (half-) infinite thickness of structures, the following expression can be established:

$$
\dot{q}^{\prime \prime}=\frac{1}{\sqrt{\pi}} \cdot \sqrt{\frac{k \cdot \rho \cdot c}{t}} \cdot\left(T_{g}-T_{a}\right)
$$

where, $\dot{q}^{\prime \prime}$ is the heat flux to surroundings, $k$ is the thermal conductivity, $\rho$ is the density and $c$ is the specific heat capacity. Eq. (9) is an expression of energy transport per unit area (flux) to the enveloping surface.

All three sizes apply to the enveloping surface material. $t$ is time in seconds. After a relatively long time, the situation becomes stationary. The time to steady state is achieved, obviously depends on the structure's thermal properties. In the steady state, the following expression is applied:

$$
\dot{q}^{\prime \prime}=\frac{k}{\delta}\left(T_{g}-T_{a}\right)
$$

where, $\delta$ is the thickness of the surrounding structures. The time at which stationary heat conduction can be assumed, i.e., the thermal penetrating time $t_{p}$ is given by:

$$
t_{p}=\frac{\delta^{2}}{4 \alpha}
$$

where, $t_{p}$ is the time at which $15 \%$ of the temperature rise in the fire room has reached the outer side of the enclosing structure. $\alpha=k / \rho \cdot c$ is the thermal diffusivity and has the unit $\mathrm{m}^{2} / \mathrm{s}$. $h_{k}$ is defined by McCaffrey et al. [2]:

$$
\begin{gathered}
h_{k}=\sqrt{\frac{k \cdot \rho \cdot c}{t}} \text { for } t<t_{p} \\
h_{k}=\frac{k}{\delta} \text { for } t \geq t_{p}
\end{gathered}
$$

If we have a combination of several different materials, we must emphasize according to the areas $\mathrm{W}$ (walls), C (ceiling) and F (floor). For example, if the walls and ceiling are made of the same material, we get the following:

$$
\begin{gathered}
h_{k}=\frac{A_{W, C}}{A_{T}} \cdot \sqrt{\frac{(k \cdot \rho \cdot c)_{W, C}}{t}}+ \\
\frac{A_{F}}{A_{T}} \cdot \sqrt{\frac{(k \cdot \rho \cdot c)_{F}}{t}}\left(t<t_{p}\right) \\
h_{k}=\frac{A_{W, C}}{A_{T}} \cdot \frac{k_{W, C}}{\delta_{W, C}}+\frac{A_{F}}{A_{T}} \cdot \frac{k_{F}}{\delta_{F}} \quad\left(t \geq t_{p}\right)
\end{gathered}
$$

where, $A_{W, C}$ is the area of surrounding walls and ceiling, $A_{F}$ is area of the floor, $k_{W, C}$ is the thermal conductivity of surrounding walls and ceiling, $k_{F}$ is the thermal conductivity of the floor, $\delta_{W, C}$ is the thickness of the walls and ceiling, respectively, $\delta_{F}$ is the thickness of the floor.

For composite materials consisting of $n$ layers, $h_{k}$ is defined as:

$$
h_{k}=\frac{1}{\sum_{i=1}^{n} \frac{1}{h_{k, i}}}
$$

Thermal properties of a selected number of materials are given in Table 1. The values are valid for ambient temperature. These properties are temperature dependent, so these materials have different properties depending on their temperature. However, the values are predicted mean values and representative for the actual temperature range. The values in Table 1 are often used in fire physical literature.

Fig. 2 shows some results obtained with the model. The figure presents a comparison between measured and calculated temperatures in the upper smoke gas layer $2.1 \mathrm{~m}$ above the floor. Furthermore, measured temperatures are depicted in $0.7 \mathrm{~m}$ and $0.2 \mathrm{~m}$ in height.

The method described above is valid when the temperature increase ranges between $20^{\circ} \mathrm{C}$ and $600^{\circ} \mathrm{C}$. Furthermore, it is assumed that there is a loss of heat through openings, as a quantity of flue gas flowing 
Table 1 Thermal properties of a selected number of materials.

\begin{tabular}{llllll}
\hline Material & $k(\mathrm{~W} / \mathrm{m} \cdot \mathrm{K})$ & $c(\mathrm{~J} / \mathrm{kg} \cdot \mathrm{K})$ & $\rho\left(\mathrm{kg} / \mathrm{m}^{3}\right)$ & $k \cdot p \cdot c\left(\mathrm{~W}^{2} \mathrm{~s} / \mathrm{m}^{4} \mathrm{~K}^{2}\right)$ & $\alpha\left(\mathrm{m}^{2} / \mathrm{s}\right)$ \\
\hline Copper & 395 & 385 & 8,920 & $1.4 \times 10^{9}$ & $1.2 \times 10^{-4}$ \\
Steel (mild) & 45.8 & 460 & 7,820 & $1.6 \times 10^{8}$ & $1.3 \times 10^{-5}$ \\
Brick (common) & 0.69 & 840 & 1,600 & $9.3 \times 10^{5}$ & $5.2 \times 10^{-7}$ \\
Concrete & $0.8-1.4$ & 880 & $1,900 \sim 2,300$ & $2 \times 10^{6}$ & $5.7 \times 10^{-7}$ \\
Lightweight concrete & 0.15 & 1,000 & 500 & $7.5 \times 10^{4}$ & $3.0 \times 10^{-7}$ \\
Glass (Pande) & 0.76 & 840 & 2,600 & $1.7 \times 10^{6}$ & $3.3 \times 10^{-7}$ \\
Gypsum plaster & 0.48 & 840 & 1,440 & $5.8 \times 10^{5}$ & $4.1 \times 10^{-7}$ \\
Fiber insulating board & 0.041 & 2,090 & 229 & $2.0 \times 10^{4}$ & $8.6 \times 10^{-8}$ \\
Aluminum & 218 & 890 & 2,700 & $5.2 \times 10^{8}$ & $9.1 \times 10^{-5}$ \\
Mineral wool, plates & 0.041 & 800 & 100 & $3.3 \times 10^{3}$ & $5.1 \times 10^{-7}$ \\
Air & 0.023 & 1,050 & 1.2 & $0.029 \times 10^{3}$ & $0.02 \times 10^{-3}$ \\
\hline
\end{tabular}



Fig. 2 Comparison between measured and calculated temperatures in the upper smoke gas layer $2.1 \mathrm{~m}$ above the floor (redrawn after) [3].

Table 2 Selected parameters.

\begin{tabular}{ll}
\hline Parameter & Definition \\
\hline$\dot{Q}$ & Energy release rate \\
$\delta$ & Thickness \\
$\rho$ & Density \\
$k$ & Thermal conductivity \\
$c$ & Specific heat capacity \\
$A_{W}$ & Area of surrounding walls \\
$A_{C}$ & Area of ceiling \\
$A_{F}$ & Area of floor \\
$A_{o}$ & Area of openings \\
$H_{o}$ & Height of opening \\
\hline
\end{tabular}

through them. In large rooms, where it takes a long time before the gas leaves the room through openings, the method cannot be used with much precision. Furthermore, the fire must be fuel-controlled in order to use the method.

\section{Discussion of Parameters}

The parameters listed in Table 2 are judged to be more or less significant with respect to their impact on the outcome from the fire model. 


\subsection{Statistical Simulations}

The statistical simulation is done with SimLab [4] coupled with the MATLAB model of the case [5]. SimLab has standard routines for different statistical distributions and can be used to make sensitivity analysis based on different methods.

\subsection{Input Parameter Variations}

The input parameters were selected to investigate a typical room. It has been decided to use a uniform distribution of most of the parameters as this is a good description if we do not know the exact values. The first three parameters are the geometry-length, width and height of the room. The next two parameters are related to ventilation - height of opening and area of opening in one facade. The next four parameters are related to the surface material in the room with its thermal properties - heat conduction, heat capacity and density and thickness of the material. The values in Table 3 are for an internal wall of concrete.

The last parameter is the energy release rate which is probably the most difficult to estimate. This is given as a normal distribution with an average value of 1,500 $\mathrm{kW}$ and a standard deviation of $150 \mathrm{~kW}$. The simulation is done for 1,800 cases. In the following, we will compare input for two types of surrounding surfaces

We compare fire scenarios in rooms surrounded by lightweight walls as well as heavy walls in order to investigate which parameters are the most significant in each case. The two types of walls are shown in Figs. 3 and 4. Furthermore, we go to a "limit" by including pure mineral wool walls to show the conditions for an adiabatic-like situation. The parameters that we differentiate during the sensitivity analysis are mainly the energy release rate, the room geometry, thermal inertia, wall thickness and height of ventilations. We investigate the temperature's sensitivity of variation in theses parameters.

\section{Results}

In Fig. 5, we can see that the simulation results from the 1,800 cases approximately show a normal distribution of the energy release rate.

If we look at the calculated distribution of the temperature in Fig. 6, it will be a normal distribution approximately. That is confirmed by looking at the probability plot in Fig. 7.

Table 3 Parameter variations (uniform).

\begin{tabular}{lll}
\hline Parameters & From & To \\
\hline Length of room & $5 \mathrm{~m}$ & $7 \mathrm{~m}$ \\
Width of room & $3.5 \mathrm{~m}$ & $4.5 \mathrm{~m}$ \\
Height of room & $2.2 \mathrm{~m}$ & $2.8 \mathrm{~m}$ \\
Height of opening & $1.5 \mathrm{~m}$ & $2.2 \mathrm{~m}$ \\
Area of opening & $2 \mathrm{~m}^{2}$ & $3 \mathrm{~m}^{2}$ \\
Heat conductivity & $0.8 \mathrm{~W} / \mathrm{mK}$ & $1.4 \mathrm{~W} / \mathrm{mK}$ \\
Heat capacity & $840 \mathrm{~J} / \mathrm{kgK}$ & $880 \mathrm{~J} / \mathrm{kgK}$ \\
Density & $1,900 \mathrm{~kg} / \mathrm{m}^{3}$ & $2,300 \mathrm{~kg} / \mathrm{m}^{3}$ \\
Thickness & $0.05 \mathrm{~m}$ & $0.1 \mathrm{~m}$ \\
\hline
\end{tabular}



(b)

Fig. 3 Rooms surrounded by lightweight walls: (a) $13 \mathrm{~mm}$ gypsum, $100 \mathrm{~mm}$ mineral wool, $13 \mathrm{~mm}$ gypsum; (b) $13 \mathrm{~mm}$ gypsum, $20 \mathrm{~mm}$ laths, $200 \mathrm{~mm}$ mineral wool, $25 \mathrm{~mm}$ air gap, wood cladding. 


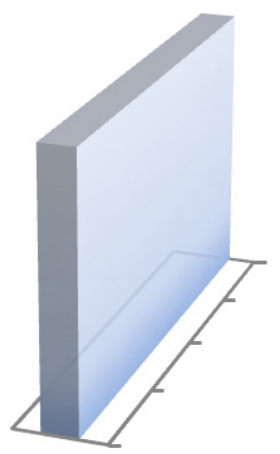

(a)



(b)

Fig. 4 Rooms surrounded by heavy walls: (a) $100 \mathrm{~mm}$ concrete; (b) $120 \mathrm{~mm}$ concrete, $150 \mathrm{~mm}$ mineral wool, $10 \mathrm{~mm}$ ventilation, $110 \mathrm{~mm}$ brick.



Fig. 5 Probability plot (normal distribution) for the energy release rate.

Figs. 8-13 present the results regarding the temperatures sensitivity with respect to variation of energy release rate and room length for the three rooms enveloped by heavy walls, lightweight walls and mineral wool walls, respectively. The temperatures are shown after $1 \mathrm{~min}$ and after $18 \mathrm{~min}$.

\subsection{Sobol Method}

The Sobol method [6] is a quantitative method that gives the percentage of the total output variance that each parameter accounts for. The method is a variance-based one to quantify the impact of uncertainties in random variables on the uncertainty in the model output. This method is more computationally expensive than the Morris method. The Sobol method for variance-based estimation is based on decomposition of the variance of a response to its variation sources. The Sobol method makes estimates 




Fig. 6 Distribution of calculated indoor temperatures after $1 \mathrm{~min}$.



Fig. 7 Probability plot (normal distribution) for the temperature after $1 \mathrm{~min}$. 


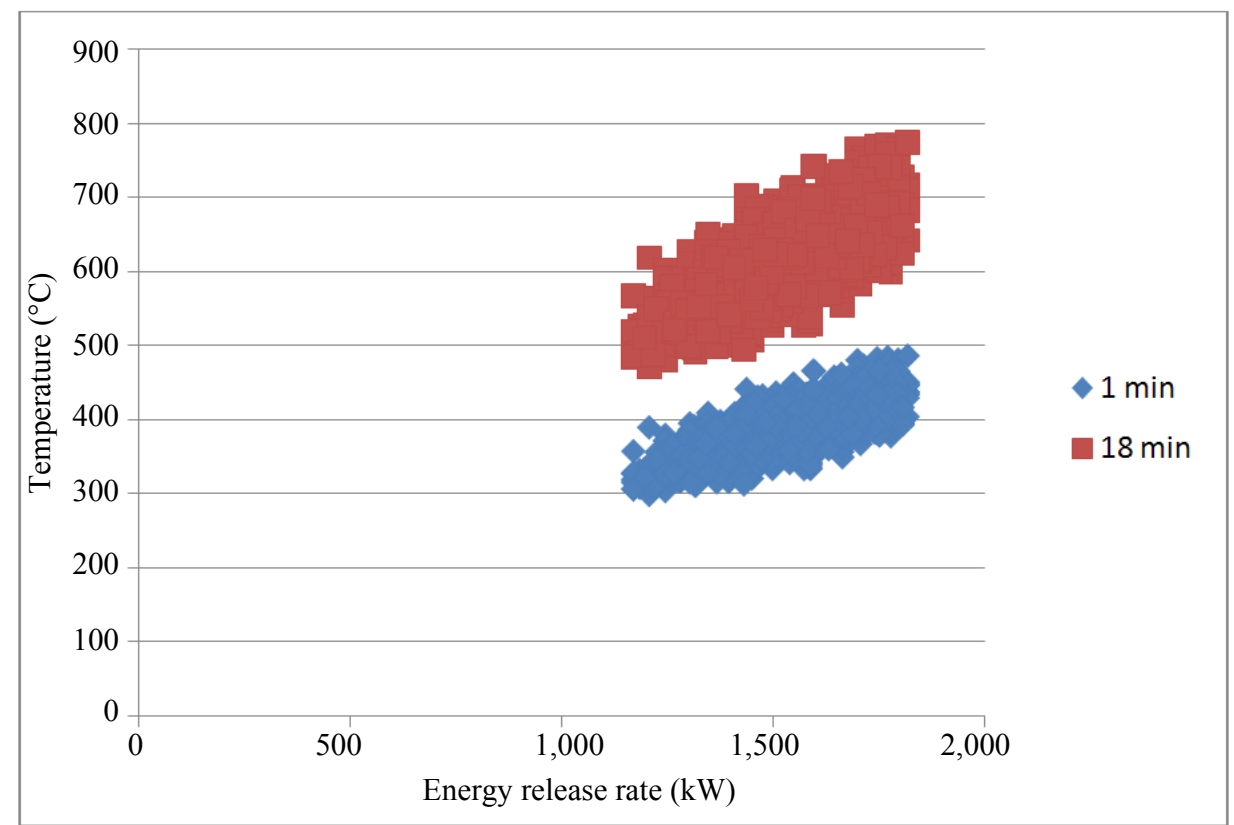

Fig. 8 Heavy walls: energy release rates versus temperatures.

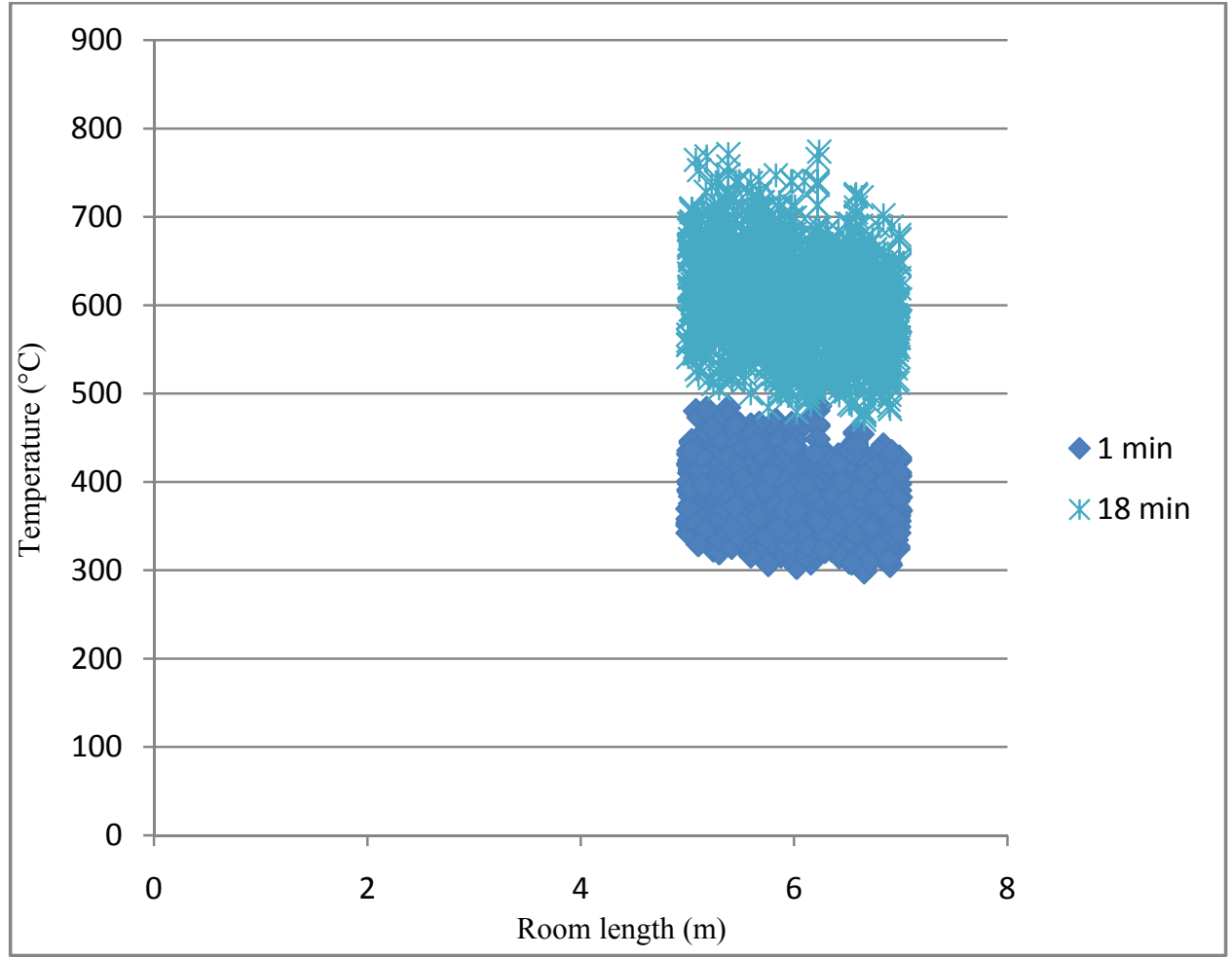

Fig. 9 Heavy walls: room length versus temperatures. 


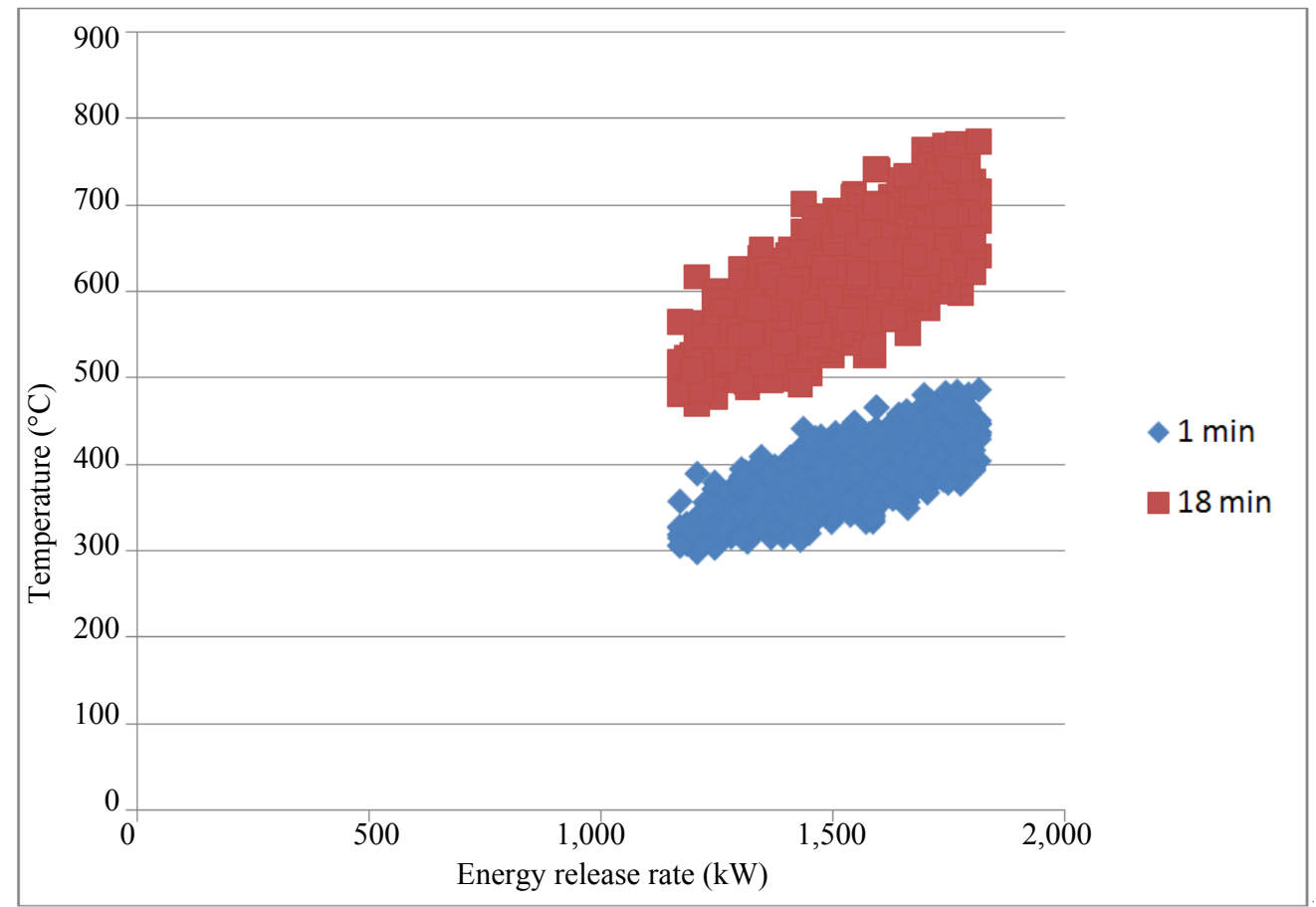

Fig. 10 Lightweight walls: energy release rates versus temperatures.

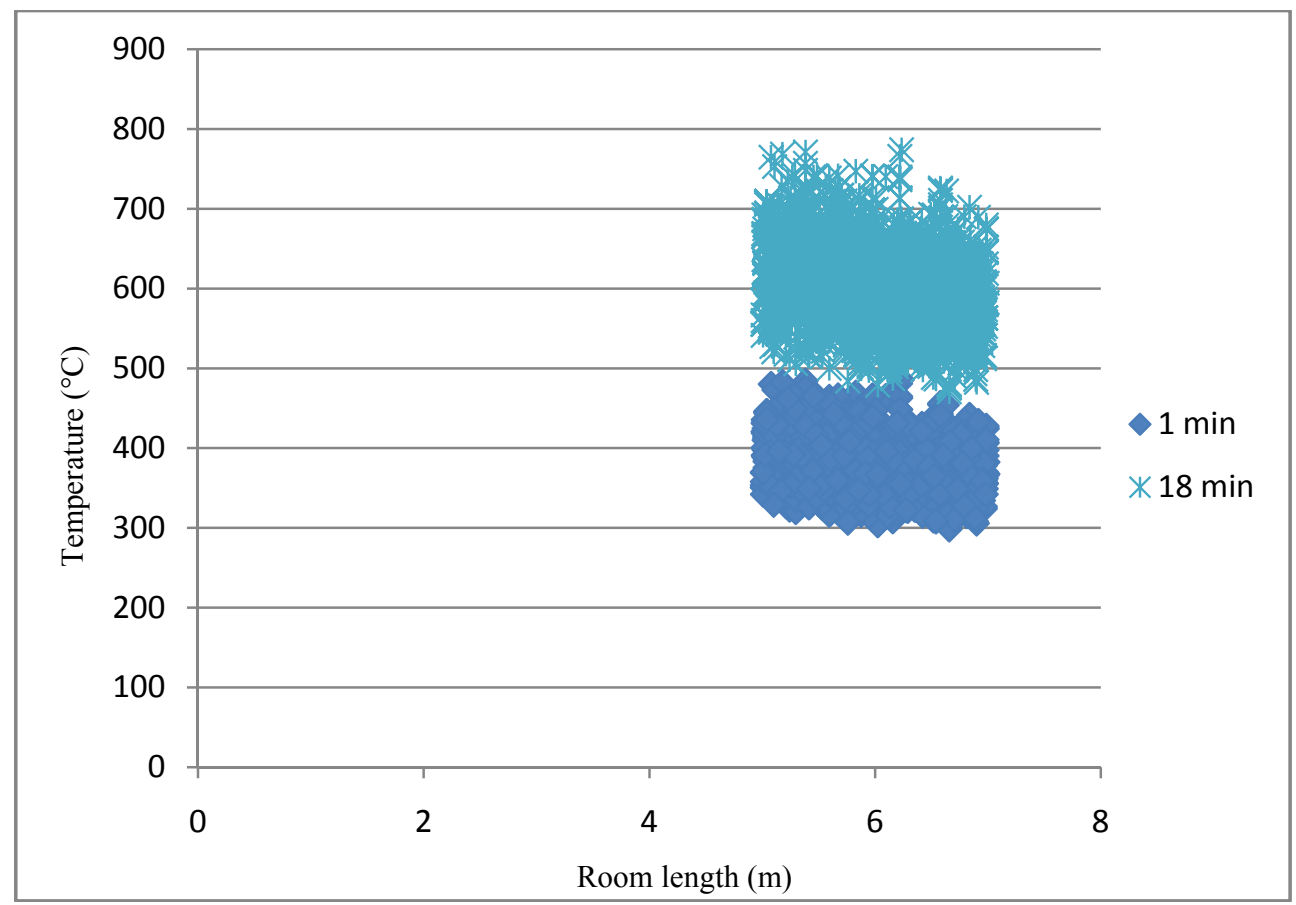

Fig. 11 Lightweight walls: room length versus temperatures. 




Fig. 12 Mineral-wool walls: energy release rates versus temperature.

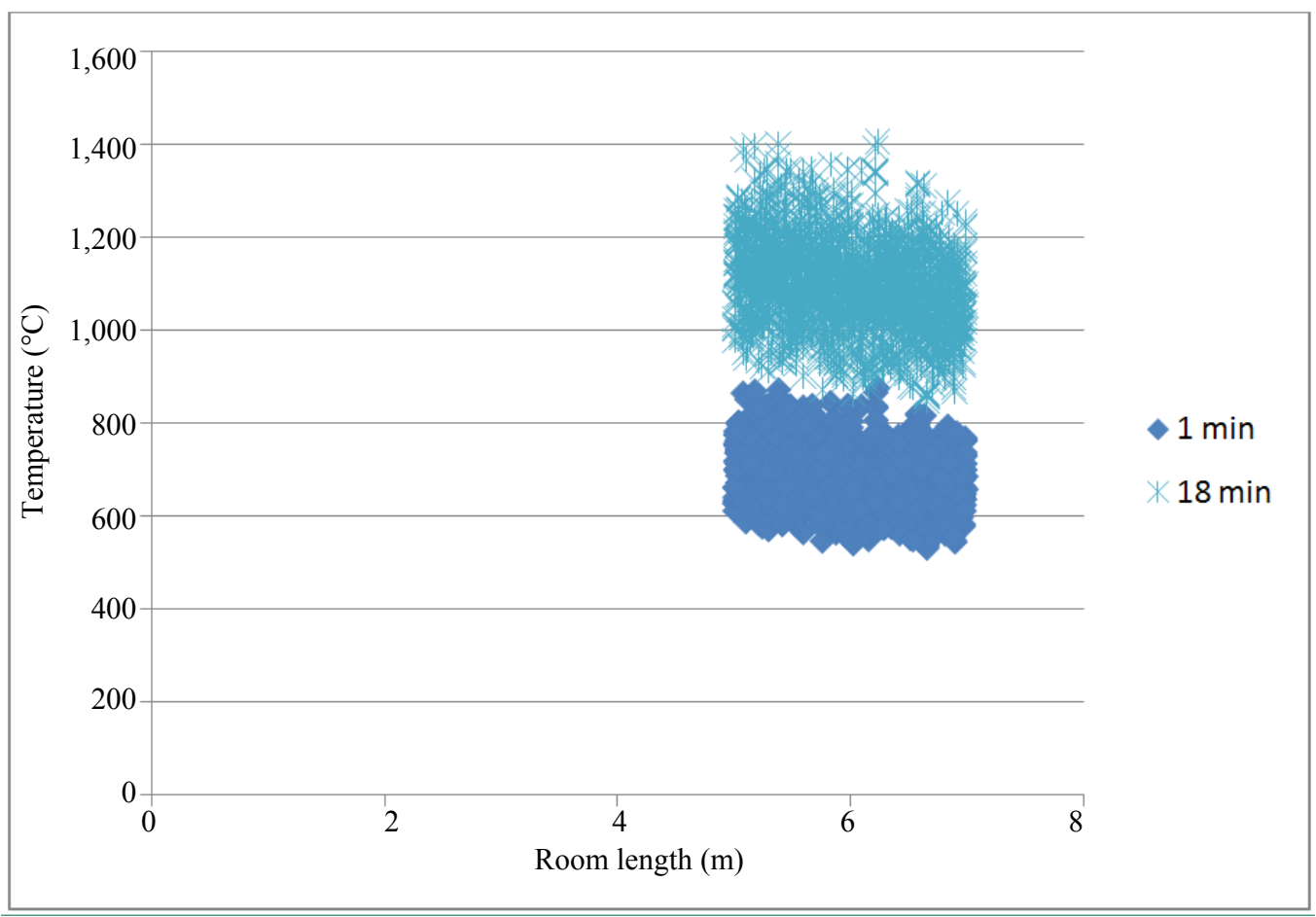

Fig. 13 Mineral-wool walls: room length versus temperature.

of first-order sensitivity indices, higher-order indices and total indices using SimLab. The first-order term represents the partial variance in the response due to the individual effects of a random variable.
The higher-order terms show the interaction between two and more variables. The total effect relates to all direct and indirect variances from other variables. 


\subsection{Sobol Analysis}

The Sobol analysis calculates the percentage of output variance that each parameter accounts for. Table 4 is for the temperature in the room with heavy constructions - concrete. It will give the same result whether we calculate after $1 \mathrm{~min}$ or $12 \mathrm{~min}$ as it is the same expression. This is only the case when we are below $t_{p}$. If we are above $t_{p}$, then the Sobol indices will change. In that case, also, the thickness of the material is important. It is seen for the case that many parameters have very little or no effect on the result. The most important parameter is the energy release rate that can explain $92 \%$ of the uncertainty in the calculated result.

The analysis is also done for all combinations of two parameters to find the combination with the largest effect. The Sobol total for pairs for the case in Table 4 has the highest value for the combination energy release rate and area of opening. That explains $96 \%$ of the uncertainty.

A similar analysis is done for the room after $18 \mathrm{~min}$ in Table 5 where the energy release rate is still the most important parameter, but now only explains $49 \%$ of the variation. The second parameter is the thickness of the surface material, which explains $43 \%$. The analysis is also done for all combinations of two parameters to find the combination with the largest effect. The Sobol total for pairs for the case in Table 5 had the highest value for the combination energy release rate and thickness of the material. That explains $88 \%$ of the uncertainty.

So, if we are going to get a more accurate prediction, we should try to get the best estimates for the energy release rate and opening area in the first phase of the fire. An incorrect estimate of the other parameters has minor effects on the results. For an improved estimate during a later phase of the fire, we also need to know the thickness of the surface material.

A similar calculation for a room with mineral wool shows that the thickness will be important much faster in the fire period.
Table 4 Sobol indices for temperature in a heavy room after $1 \mathrm{~min}$ and $12 \mathrm{~min}$.

\begin{tabular}{lll}
\hline Parameters & Sobol first & Sobol total \\
\hline Length of room & 0.0194 & 0.0173 \\
Width of room & 0.0049 & 0.0072 \\
Height of room & -0.0093 & 0.0036 \\
Height of opening & 0.0107 & 0.0087 \\
Area of opening & 0.0400 & 0.0358 \\
Heat conductivity & -0.0088 & 0.0176 \\
Heat capacity & 0.0004 & 0.0001 \\
Density & 0.0014 & 0.0021 \\
Thickness & 0 & 0 \\
Energy release rate & 0.9057 & 0.9183 \\
\hline
\end{tabular}

Table 5 Sobol indices for temperature in the room after 18 min.

\begin{tabular}{lll}
\hline Parameters & Sobol first & Sobol total \\
\hline Length of room & 0.0118 & 0.0095 \\
Width of room & 0.0005 & 0.0038 \\
Height of room & -0.0061 & 0.0020 \\
Height of opening & 0.0053 & 0.0048 \\
Area of opening & 0.0208 & 0.0189 \\
Heat conductivity & 0.0069 & 0.2112 \\
Heat capacity & 0.0001 & 0.0001 \\
Density & 0.0131 & 0.1221 \\
Thickness & 0.0533 & 0.4342 \\
Energy release rate & 0.4721 & 0.4917 \\
\hline
\end{tabular}

\section{Conclusions}

A method to do a sensitivity analysis of parameters used in a simplified fire model for temperature estimates in the upper smoke layer during a fire is discussed. Fire scenarios in rooms surrounded by lightweight walls as well as heavy walls are compared in order to investigate which parameters are the most significant. We utilize the Sobol method, which is a quantitative method that gives the percentage of total output variance which each parameter accounts for.

The energy release rate is found to be the most important parameter and can explain $92 \%$ of the uncertainty in the results for the period before thermal penetration $\left(t_{P}\right)$ has occurred. The analysis is done for all combinations of two parameters as well. The Sobol total for pairs had the highest value for the combination energy release rate and area of opening. That explains $96 \%$ of the uncertainty. After thermal penetration, the 
energy release rate is still the most important parameter, but now only explains $49 \%$ of the variation. The second parameter is the thickness of the surface material, which explains $43 \%$. The analysis is done for all combinations of two parameters in order to find the combination with the largest effect. The Sobol total for pairs had the highest value for the combination energy release rate and the thickness of the material, which explains $88 \%$ of the uncertainty.

The proposed method is found to be a proper way to estimate which parameters are most sensitive for the results obtained in a simplified fire model. Sensitivity analyses have also been used in other areas [7-9].

\section{References}

[1] Sørensen, L. S. 2014. Fire-Safety Engineering and Performance-Based Codes. Denmark: Polyteknisk Forlag.

[2] McCaffrey, B. J., Quintiere, J. G., and Harkleroad, M. F. 1981. "Estimation Room Fire Temperatures and the Likelihood of Flashover Using Fire Test Data Correlations." Fire Technology 17 (2): 98-119.

[3] Karlsson, B., and Quintiere, J. G. 2000. Enclosure Fire Dynamics. London: CRS Press.
[4] European Commission. 2014. SIMLAB Version 3.2.9. European Commission. Accessed November 16, 2014. http://simlab.jrc.ec.europe.eu.

[5] The MathWorks, Inc. 2015. MATLAB Version 7 with SIMULINK. The MathWorks, Inc. Accessed January 12, 2015. http://www.mathworks.com.

[6] Saltelli, A., Tarantola, S., Campolongo, F., and Ratto, M. 2004. Sensitivity Analysis in Practice: A Guide to Assessing Scientific Models. USA: Wiley.

[7] Kleijnen, J. P. C. 1995. "Sensitivity Analysis and Related Analyses-A Survey of Statistical Techniques." Presented at International symposium SAMO95-Theory and Applications of Sensitivity Analysis of Model Output in Computer Simulations, Italy.

[8] Nielsen, A., Møller, E. B., Rasmussen, T. V., and Hansen, E. J. P. 2012. "Use of Sensitivity Analysis to Evaluate Hygrothermal Conditions in Solid Brick Walls with Interior Insulation." In Proceedings of the 5th IBPC (International Building Physics Conference): The Role of Building Physics in Resolving Carbon Reduction Challenge and Promoting Human Health in Buildings, 377-84.

[9] Nielsen, A., Wittchen, K. B., and Bertelsen, N. H. 2014. "Sensitivity Analysis of the Energy Demand of Existing Buildings Based on the Danish Building and Dwelling Register." In Proceedings of NSB 2014: 10th Nordic Symposium on Building Physics, 271-8. 